\title{
Portación nasal de Staphylococcus aureus en una cohorte de niños con cáncer
}

\author{
M. Teresa Dossi C., Guillermo Zepeda F. y Walter Ledermann D.
}

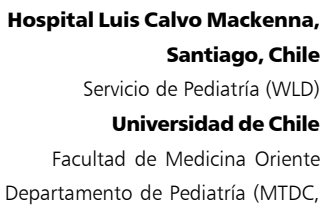

GZF)

Recibido: 4 septiembre 2006 Aceptado: 27 diciembre 2006

Correspondencia a:

Walter Ledermann Dehnhardt oncemayor@gmail.com

\section{Nasal carriage of Staphylococcus aureus in a cohort of children with cancer}

According to medical literature, nasal carriage of Staphylococcus aureus in pediatric age in the general population ranges between 10 and $40 \%$, not being clearly established in children with cancer. In order to determine the prevalence in this group and its possible increment through successive hospitalizations, we studied the Calvo Mackenna's oncologic patients below 19 years of age, with a series of nasal cultures. We took four samples for each patient in a period of one year. Of 80 patients initially included, 10 died, 9 returned to their city of origin before the end of the study and in 8 cases it was not possible to complete the follow-up. Prevalence of nasal carriage was $21.2 \%$ (17/80). From 17 children colonized, 14 lost this condition, 2 abandoned the follow-up and only 1 was positive at the end of the study. Incidence of hospitalization due to febrile neutropenia was $28.7 \%(23 / 80)$ and the relation between nasal carriage and the number of these events was not significant $(\mathrm{p}=0.59)$. The relation between the number of hospitalizations and an increment in nasal colonization $(\mathrm{p}=0,80)$ was also not significant. In conclusion, nasal carriage of Staphylococcus aureus in children with cancer is similar to carriage described for healthy children. Carriage would be a transitory condition, variable through time for each patient, without relation to a greater number of febril neutropenia episodes.

Key words: Staphylococcus aureus, carriage, neutropenia, cancer.

Palabras claves: Staphylococcus aureus, portación, neutropenia, cáncer.

\section{Introducción}

D esde 1988, se han registrado en Chile más de cuatro mil pacientes pediátricos con cáncer, los que han recibido tratamiento sistemático y completo para su patología a través del Programa Infantil Nacional de Drogas Antineoplásicas (PINDA), con promedios de curación de $\sim 65 \%^{1,2}$. Dentro de este tratamiento destaca la quimioterapia como arma terapéutica importante, procedimiento que unido a sus innumerables beneficios, presenta complicaciones por su toxicidad sobre el ciclo de todas las células del organismo, en especial sobre aquellas de gran multiplicación, como son las del sistema hematopoyético ${ }^{3}$. De particular riesgo es la neutropenia, cuya importancia radica en la relación estrecha que existe entre el recuento absoluto de neutrófilos (RAN) y la probabilidad de infección, que ocurre en forma casi invariable cuando el RAN es inferior a 100 células por $\mathrm{mm}^{3} .^{3}$.

En Chile, no se ha establecido en forma precisa la frecuencia de neutropenia febril (NF) en pacientes oncológicos, pero se sabe, a través del PINDA, que en seis hospitales de Santiago se atiende un promedio de 400 episodios de NF por año ${ }^{4}$, en tanto que 30 a $35 \%$ serían de etiología probablemente bacteriana, aunque no demostrada en exámenes de laboratorio ${ }^{5,6}$. Dentro de las etiologías bacterianas se describió a Staphylococcus aureus como causante de $16,7 \%$ (sobre un total de 707 cepas aisladas) de las infecciones bacterianas demostradas en este grupo de pacientes ${ }^{7}$.

Esta bacteria puede estar presente como comensal en diferentes regiones del organismo, lo que ocurriría con una frecuencia variable entre 10 y $40 \%$ de la población pediátrica en general, siendo el vestíbulo nasal el sitio más común de colonización y una de las puertas de entrada hacia el torrente sanguíneo ${ }^{8}$.

Todos los estudios nacionales de portación nasal intrahospitalaria se refieren al personal de la salud, incluyendo también alumnos en práctica; $\mathrm{M}$. Cifuentes ${ }^{9}$ encontró $36,8 \%$ de portadores entre estudiantes de medicina. En cuanto a pacientes infantiles, el único antecedente es el estudio de P. Madrid et $\mathrm{al}^{10}$, quienes encontraron $34,6 \%$ de portación en una cohorte de 101 niños internados en el Hospital Luis Calvo Mackenna (HLCM), tras uno y tres días de hospitalización.

No se ha establecido en la literatura médica si la portación nasal de esta bacteria es más frecuente en 
niños oncológicos que en la población general, ni si existe una mayor frecuencia de infecciones invasoras por este agente entre los portadores.

En este estudio intentamos, como primer objetivo, determinar la prevalencia de portación nasal de $S$. aureus en niños con cáncer. En segundo término, investigar la existencia de una mayor probabilidad de colonización nasal entre estos niños, a medida que va aumentando el número de sus hospitalizaciones en el curso de un año.

\section{Pacientes y Métodos}

Estudio prospectivo, descriptivo, que incluyó a todos los pacientes con edad igual o inferior a 19 años, que se controlaban ambulatoriamente en la policlínica de Oncología del HLCM, con diagnóstico de cáncer, en cualquier etapa de quimioterapia, o sometidos a trasplante de precursores hematopoyéticos. No se excluyó paciente alguno.

Para obtener la prevalencia basal de portación nasal durante dos meses, de octubre a diciembre de 2004, se reclutaron todos los pacientes controlados en Oncología, a los cuales, previo consentimiento, se les tomó cultivo nasal para detectar la colonización por $S$. aureus, mediante técnica estéril, introduciendo una tórula de algodón al tercio anterior de cada una de ambas fosas nasales. Las muestras fueron procesadas de inmediato en el laboratorio de Microbiología, sembrándose en agar sangre e incubándose a $37^{\circ} \mathrm{C}$ por 24 a 48 horas.

Durante un período de 12 meses (enero del 2005 a enero del 2006), se siguió a esta cohorte de niños oncológicos, con diagnóstico establecido previamente, registrándose los siguientes datos:

- Cultivos nasales para $S$. aureus, con un total de cuatro muestras por paciente, separadas por un tiempo mínimo de un mes y máximo de cuatro meses, tomadas en la policlínica de Oncología.

- Número de hospitalizaciones por NF durante el período, comprendiendo dentro de éstas las hospitalizaciones por infecciones bacterianas invasoras (IBI).

- Etiología de éstas, determinada por hemocultivos periféricos y/o de catéter venoso central, urocultivos, cultivos de LCR, articular y otros.

No se consideraron hospitalizaciones por otras causas, como quimioterapia, cambio de catéter, etc.

\section{Definiciones}

Fiebre: una temperatura axilar igual o mayor a 38,5 ${ }^{\circ} \mathrm{C}$, en una medición, o igual o mayor a $38^{\circ} \mathrm{C}$ en dos mediciones separadas por una hora.
Neutropenia grave: RAN de 500 células por $\mathrm{mm}^{3} \mathrm{o}$ menos.

Infección bacteriana demostrada: cumplimiento de uno o ambos de los siguientes criterios: bacteriemia, es decir, uno o más hemocultivos positivos para alguna especie bacteriana, (dos o más cultivos positivos en caso de aislarse Staphylococcus coagulasa-negativa), y cultivos positivos de líquidos normalmente estériles, como LCR, orina, articular u otros.

Análisis estadístico. Se estableció la prevalencia de portación nasal con un nivel de confianza de $95 \%$ y un grado de error de $8 \%$, presumiendo una prevalencia de $50 \%$, que exigiría tamaño máximo de muestra. Como éste podría ser inferior a 150 casos y la disponibilidad de pacientes no superó los 80 , se decidió considerar esta muestra como estudio piloto. Como medida secundaria de los resultados, por el número de casos susceptibles de reunir y el tiempo limitado de investigación, se efectuó una comparación en términos de la frecuencia de presentación de infección sistémica en pacientes portadores nasales de $S$. aureus y pacientes no portadores. Las variables estudiadas fueron comparadas como proporciones, usando la prueba de Fisher, con un error $\alpha$ de $5 \%$.

Tanto este protocolo de estudio como el consentimiento informado que debían otorgar los pacientes para ingresar a él, fueron aprobados por el Comité de Ética de la Investigación del HLCM.

\section{Resultados}

En total se logró reclutar 80 pacientes, de los cuales 49 eran de sexo masculino y 31 de sexo femenino. El promedio de edad fue de 9,28 años, con un rango de 5 meses a 19 años.

Sus diagnósticos eran leucemia linfoblástica aguda (LLA) en 42 de ellos, osteosarcoma en 13, rabdomiosarcoma en 5, leucemia mieloide aguda (LMA) en 3, neuroblastoma en 3, linfoma de Hodgkin en 3, tumor neuro-ectodérmico periférico (PNET) en 3, tumor de Wilms en 2, sarcoma de Ewing 1, sarcoma hepático 1, meduloblastoma 1 , hepatoblastoma 1 , sarcoma de partes blandas 1 y leucemia mieloide crónica (LMC) 1 .

El número inicial se fue reduciendo en cada toma de muestra durante el seguimiento. Las causas de abandono del estudio fueron: fallecimiento en 10 casos $(12,5 \%)$; regreso a su lugar de origen, discontinuando su control en el HLCM, 9 niños (11\%); falta de seguimiento por razones varias en 8 niños (10\%) (Figura 1).

Dinámica de la portación. (Tabla 1). A cada paciente se le tomó la muestra 1 (M1) a su ingreso al estudio, obteniendo con ella una prevalencia de portación nasal de $S$. aureus de 21,2\% (17/80), (IC 95\%: 


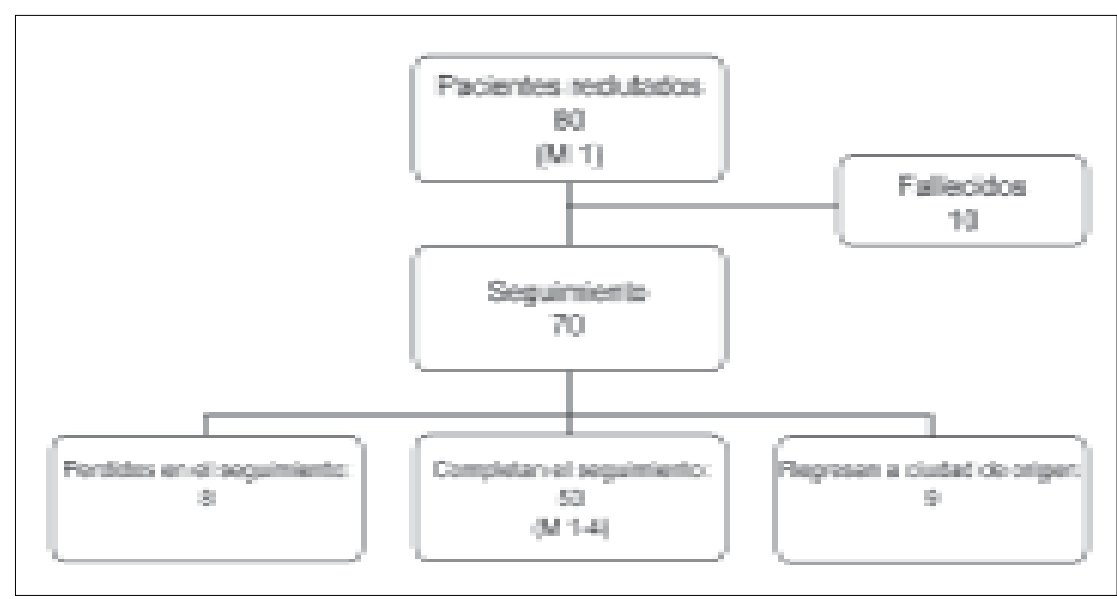

Figura 1. Flujograma del estudio. $M$ 1: muestra basal. M 1-4: muestras 1-4.

\begin{tabular}{|c|c|c|c|c|c|}
\hline Muestra & $\begin{array}{c}\text { n } \\
\text { pacientes }\end{array}$ & $\begin{array}{l}\text { Mantiene } \\
\text { portación }\end{array}$ & $\begin{array}{l}\text { Adquiere } \\
\text { portación }\end{array}$ & $\begin{array}{c}\text { Pierde } \\
\text { portación }\end{array}$ & $\begin{array}{c}\text { Total } \\
\text { portadores }\end{array}$ \\
\hline M 1 & 80 & - & - & - & 17 \\
\hline M2 & 63 & 4 & 5 & 11 & 9 \\
\hline M3 & 58 & 5 & 2 & 4 & 7 \\
\hline M4 & 53 & 2 & 3 & 5 & 5 \\
\hline
\end{tabular}

Tabla 2. Portación nasal global de Staphylococcus aureus en el seguimiento de un año en $\mathbf{5 3}$ niños con cáncer

\begin{tabular}{|c|c|c|c|c|c|}
\hline \multirow[b]{2}{*}{ Condición inicial } & \multirow[b]{2}{*}{ n de niños } & \multicolumn{4}{|c|}{ Número de portadores nasales } \\
\hline & & M 1 & M2 & M3 & M4 \\
\hline Portadores & 15 & 15 & 4 & 3 & 1 \\
\hline No portadores & 38 & 0 & 5 & 4 & 4 \\
\hline Total (\%) & $53(100)$ & $15(28,3)$ & $9(16,9)$ & $7(13,2)$ & $5(9,4)$ \\
\hline
\end{tabular}

\begin{tabular}{|c|c|c|c|c|}
\hline $\begin{array}{l}\text { n de } \\
\text { hospitalizaciones }\end{array}$ & Portadores & No portadores & $\begin{array}{l}\text { Total } \\
\text { niños }\end{array}$ & $\begin{array}{c}\text { Total } \\
\text { hospitalizados }\end{array}$ \\
\hline Ninguna & 12 & 20 & 32 & 0 \\
\hline Una & 5 & 8 & 13 & 13 \\
\hline Dos & 3 & 4 & 7 & 7 \\
\hline Tres & 0 & 1 & 1 & 1 \\
\hline Total niños & 20 & 33 & 53 & 21 \\
\hline
\end{tabular}

13,2-32,1). Durante el seguimiento, para la muestra 2 (M2) se logró reclutar a 63 pacientes, observándose que de los 17 portadores con M1, siguieron positivos 4 , en tanto que de los 13 restantes, uno abandonó el estudio, otro falleció y 11 se negativizaron (de éstos, 5 habían recibido tratamiento antimicrobiano, 4 de ellos por NF). En cambio, de los pacientes que fueron negativos con M1, 5 se mostraron colonizados en M2, lo que arroja un total de 9 portadores en M2.

Para la muestra 3 (M3) se logró contactar 58 pacientes. De los 9 que aparecían colonizados con M2, 5 permanecieron positivos y 4 dejaron de ser portadores. De estos 4 negativizados, 3 habían recibido tratamiento antimicrobiano por NF. De los pacientes no colonizados en $\mathrm{M} 2$, se hicieron portadores 2, quedando un total de 7 portadores para M3.

Para la muestra 4 (M4) se contactaron 53 de los 58 pacientes anteriores. De los 7 positivos con M3, 2 mantuvieron esta condición y 5 se negativizaron, habiendo dos de éstos recibido tratamiento antimicrobiano. De los pacientes negativos con M3, aparecieron ahora positivos 3 , quedando un total de 5 positivos para M4.

La incidencia de portación nasal de $S$. aureus en el período de tiempo del estudio fue de $28,7 \%$, ya que, en alguna o varias de las cuatro muestras, 23 de los 80 pacientes inicialmente enrolados tuvieron resultados positivos. La incidencia de hospitalizaciones por NF durante el año 2005 para este grupo de 80 pacientes fue del 28,7\% (23/80) (IC 95\% 23,4-34,2).

Trascendencia de la colonización nasal. No se aisló $S$. aureus como agente causal de IBI de sitio estéril alguno. En 7\% de los pacientes (6/80) hubo aislamiento de agente etiológico, correspondiendo 2 a Escherichia coli, 2 a Staphylococcus coagulasa-negativa y 2 a Pseudomonas aeruginosa. Sólo 53 pacientes completaron el seguimiento, incluyendo 15 de los 17 inicialmente positivos, de los cuales sólo uno terminó positivo al cabo del año, y 38 de los 63 inicialmente negativos, de los que 4 terminaron colonizados. (Tabla 2). En esta cohorte de 53 pacientes hubo 21 hospitalizaciones por NF $(39,6 \%)$. De estos 21 niños hospitalizados, sólo 8 estuvieron alguna vez colonizados por $S$. aureus. No se encontró relación entre el antecedente de colonización nasal y frecuencia de hospitalizaciones por NF $(p=0,59)$. En cuanto a la relación entre número de hospitalizaciones por NF y la adquisición de colonización nasal, entre los 53 pacientes con seguimiento completo, hubo 32 que nunca se hospitalizaron por NF, de los cuales 12 estuvieron alguna vez colonizados y 20 nunca; por otra parte, de los 21 que se hospitalizaron por esta causa, 13 nunca fueron portadores. De esta manera, no pudo establecerse una relación entre el número de 
hospitalizaciones y la frecuencia de portación nasal $(\mathrm{p}=80)($ Tabla 3$)$.

Hubo sólo un paciente con aislamiento de S. aureus en las cuatro muestras, niño que tuvo sólo una hospitalización por NF de alto riesgo durante el año de observación, ocasión en que no se encontró ni ésta ni otra bacteria como agente etiológico. Otro paciente con tres muestras nasales positivas en forma consecutiva no registró hospitalizaciones por NF.

\section{Discusión}

Analizando los resultados, vemos que la incidencia de portación nasal de $S$. aureus en los pacientes oncológicos se sitúa entre los amplios márgenes descritos en la literatura médica para la población general. En una comparación más acotada, con el mencionado estudio de P. Madrid, que mostró 34,6\% de portación en 101 niños internados en la misma institución diez años antes, de los cuales ninguno tenía cáncer, la portación de nuestros pacientes resulta incluso menor. Sin embargo, dicho estudio fue sólo una instantánea, reflejando la situación al ingreso y sin un posterior seguimiento, falencia importante, pues esta condición es variable en el tiempo y diferente para cada paciente. Esta variabilidad pudiera explicarse por el uso de medicamentos anti-estafilocóccicos en cada hospitalización por NF, que pondría término a la portación, pero faltan estudios que avalen esta hipótesis.

En total, de los 17 pacientes positivos en M1, 10 dejaron de ser portadores en coincidencia con tratamiento antimicrobiano entre una muestra y otra. En 9 casos, en que las cepas portadas eran sensibles a meticilina, el tratamiento comprendió como fármaco anti-estafilocóccico únicamente cloxacilina, que está incluida en los dos esquemas usados en NF en el hospital: asociada a cefotaxima para los episodios de bajo riesgo, y asociada a ceftazidima y amikacina en los episodios con alto riesgo de infección invasora. En el caso restante, cuya cepa portada era resistente a meticilina, se incluyó vancomicina en el esquema.

Nuestros resultados muestran que no existiría una mayor frecuencia de hospitalizaciones por NF entre los niños que alguna vez fueron portadores, en comparación con los que nunca lo fueron, situación que podría deberse tanto a ser la portación una condición transitoria en el tiempo, como a no ser $S$. aureus un agente causal frecuente de $\mathrm{NF}^{11}$. Así, el número de hospitalizaciones no influiría en la condición de portador, ni existiría relación directa entre el número de hospitalizaciones y la portación nasal. De hecho, los pacientes que más veces se hospitalizaron por NF estuvieron menos colonizados que aquellos que nunca se hospitalizaron.

Como no se encontró IBI por esta especie bacteriana en alguno de los pacientes durante el año de seguimiento, no pudo comprobarse si la cepa aislada de vestíbulo nasal era la misma causante del cuadro sistémico.

\section{Agradecimientos}

A todas las personas que contribuyeron a la realización de este laborioso y arduo trabajo, en especial al personal del Laboratorio de Microbiología, por su colaboración en el procesamiento de las muestras; a Gastón Duffau por su ayuda en el diseño y análisis estadístico de esta investigación; y a M. Elena Santolaya por su orientación y apoyo.

\section{Resumen}

Para determinar si la prevalencia de portación nasal de Staphylococcus aureus en niños cáncer es mayor que en la población general pediátrica, donde fluctuaría entre 10 y $40 \%$, así como demostrar si esta colonización va a aumentando con el número de hospitalizaciones, se efectuó un seguimiento durante un año, mediante cuatro cultivos nasales seriados, a todos los pacientes oncológicos bajo 19 años de edad, en control en la Unidad de Oncología del Hospital Luis Calvo Mackenna. De 80 pacientes inicialmente incluidos, 10 fallecieron, 9 regresaron a su lugar de origen y en 8 no fue posible completar el seguimiento. La prevalencia inicial de portación fue de $21,2 \%$ (17/80). De los 17 niños portadores, 14 perdieron su condición, 2 abandonaron el seguimiento y sólo uno era positivo al final del estudio. La incidencia de hospitalizaciones por episodios de neutropenia febril fue de 28,7\% (23/80). La relación entre portación y el número de estas hospitalizaciones no fue significativa $(p=0,59)$, ni tampoco la relación entre mayor número de hospitalizaciones e incremento de la colonización $(p=0,80)$. En conclusión, la portación nasal de $S$. aureus en niños oncológicos es similar a la descrita para niños sanos; transitoria y variable en el tiempo, no se relaciona con un mayor número de hospitalizaciones. 


\section{Referencias}

1.- Vargas L. Avances en el tratamiento del cáncer infantil en Chile. Diez años del Programa Infantil Nacional de Drogas Antineoplásicas (PINDA). Rev Chil Pediatr 1998; 69: 270-5.

2.- Vargas L. Cáncer en pediatría. Aspectos generales. Rev Chil Pediatr 2000; 71 : 283-95.

3.- Bodey G P, Buckley M, Sathe Y S, Freirech E J. Quantitative relationships between circulating leukocytes and infection in patients with acute leukemia. Ann Intern Med 1996; 64: 328-40.

4.- Santolaya M E, Álvarez A M, Avilés C, Becker A, Cofré J, Cumsille M, et al. Early hospital discharge followed by outpatient management versus continued hospitalization of children with cancer, fever and neutropenia at low risk for invasive bacterial infection. J Clin Oncol 2004; 22: 3784-9.

5.- Santolaya M E, Álvarez A M, Becker A, Cofré J, Enríquez N, O’Ryan M, et al. Prospective, multicenter evaluation of risk factors associated with invasive bacterial infection in children with cancer, neutropenia and fever. J Clin Oncol 2001; 19: 3415-21.

6.- Santolaya M E. Neutropenia febril en el niño con cáncer. Conceptos actuales sobre criterios de riesgo y manejo selectivo. Rev Méd Chile 2001; 129: 1449-54.

7.- Payá E, Álvarez A M, Avilés C, Cofré J, Enríquez N, Salgado C, et al. Agentes causantes de infecciones del torrente circulatorio en niños con cáncer en cinco hospitales de Santiago (1994-1998). Rev Méd Chile 2001; 129: 1297-304.

8.- Anjali J, Daum R. Staphylococcal infections in children. Part I. Pediatr Rev 1999; 20: 183-91.

9.- Cifuentes M, Prado V, Ojeda A. Prevalencia de portación de Staphylococcus aureus y Staphylococcus aureus meticilino resistente en estudiantes de medicina y población general. Rev Chil Infect 1998; 15: 161-9.

10.- Madrid P, Hering E, Kägi M, Díaz J M, Molina $\mathrm{P}$, et al. Incidencia de la portación nasal de Staphylococcus aureus y de su resistencia a cloxacilina en pacientes con uno y tres días de hospitalización. Calvomackenna 1996; 2: 53-6.

11.- PINDA. Ministerio de Salud, Chile 1994. Recomendaciones para el diagnóstico tratamiento y prevención de las infecciones en los pacientes oncológicos en edad pediátrica. Rev Chil Infect 1994; 11: 11926. 